Supporting Information

\title{
Direct Color Printing with an Electron Beam
}

Soroosh Daqiqeh Rezaei ${ }^{1,2,3, *}$, Jinfa Ho ${ }^{1}$, Tao Wang ${ }^{1,4}$, Seeram Ramakrishna ${ }^{2}$, and Joel K. W. Yang ${ }^{1,3, *}$

${ }^{1}$ Nanofabrication Department, Institute of Materials Research and Engineering (IMRE), Agency for Science, Technology and Research (A*STAR), 2 Fusionopolis Way, 138634, Singapore

${ }^{2}$ Department of Mechanical Engineering, Faculty of Engineering, National University of Singapore, 117575, Singapore

${ }^{3}$ Pillar of Engineering Product Development, Singapore University of Technology and Design, 8 Somapah Road, 487372, Singapore

${ }^{4}$ Institute of Functional Nano and Soft Materials (FUNSOM), Jiangsu Key Laboratory for Carbon-Based Functional Materials and Devices, Soochow University, Suzhou, 215123, China

*Correspondence: soroosh@u.nus.edu; joel_yang@sutd.edu.sg 
a Initial thickness $h=168 \mathrm{~nm}$

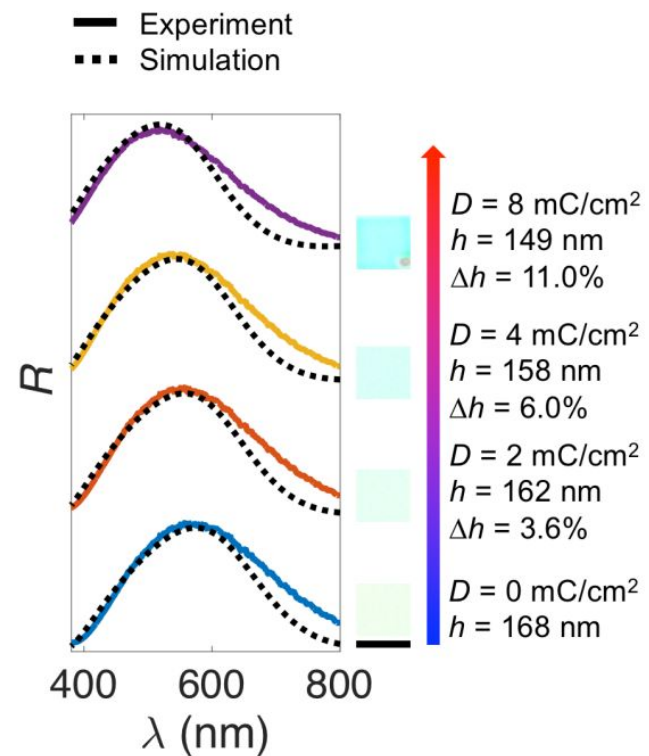

b Initial thickness $h=500 \mathrm{~nm}$

- Experiment

... Simulation

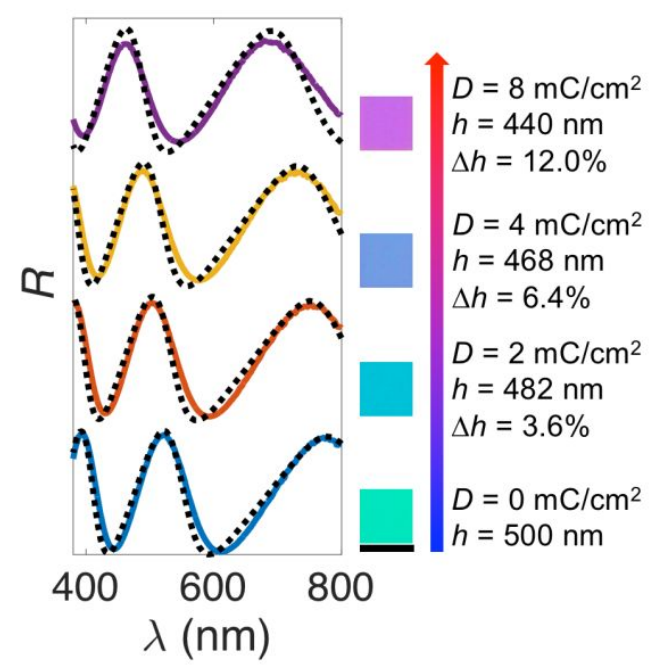

Figure S1. Reflectance spectra and the corresponding optical micrograph, exposure duration, and fractional shrinkage for FP cavities with initial thicknesses of (a) $h=168 \mathrm{~nm}$ and (b) $h=$ $500 \mathrm{~nm}$. Optical micrographs are captured using a $20 \times$ objective lens $(\mathrm{NA}=0.45)$ and scale bars represent $10 \mu \mathrm{m}$. 
Experimental and simulated reflectance spectra with the corresponding colors

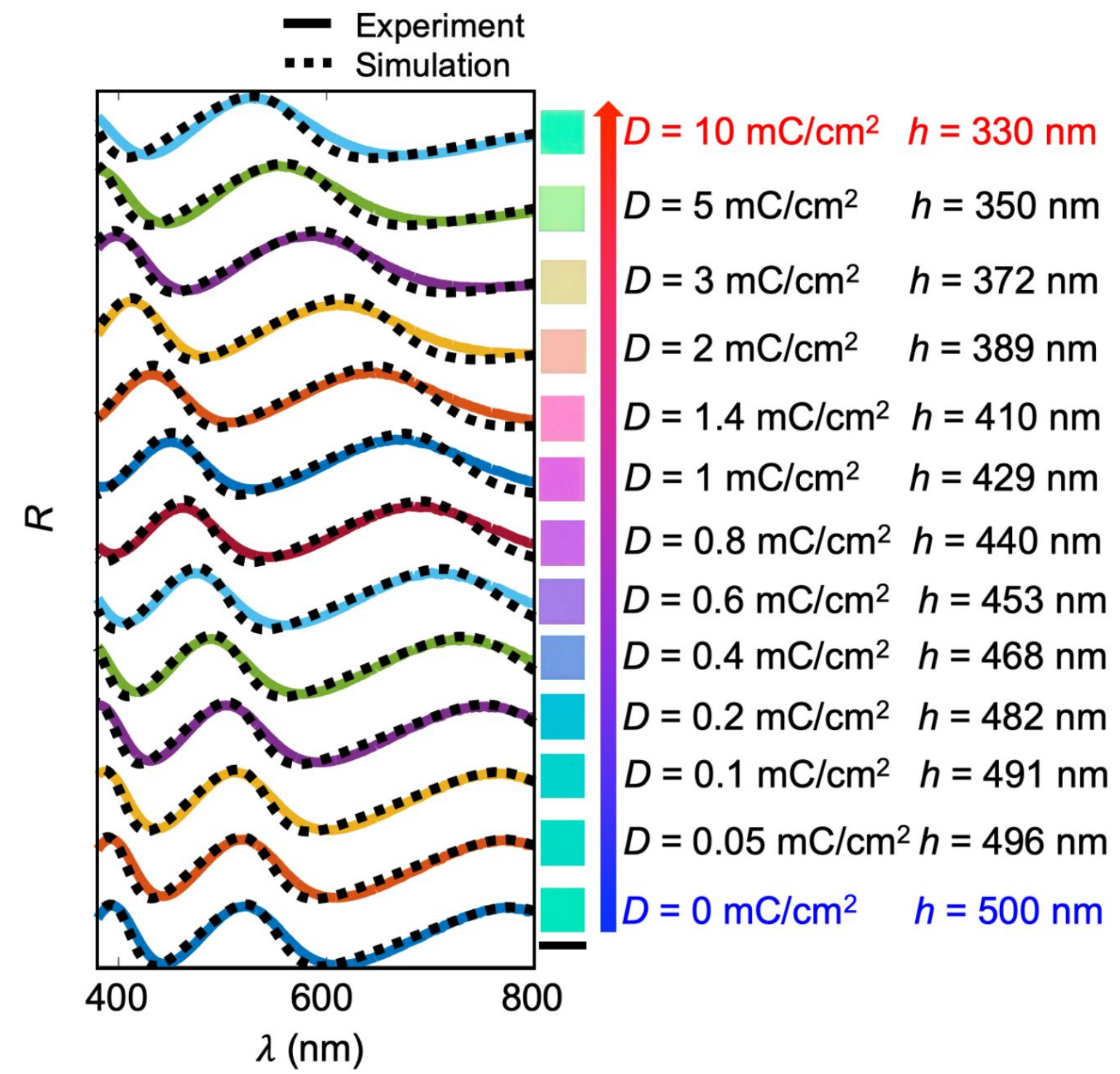

Figure S2. Experimental and simulated reflectance spectra of the square arrays exposed to Ebeam. The corresponding optical micrograph, exposure duration, and thickness are depicted next to each spectrum. Scale bar represents $10 \mu \mathrm{m}$. Optical micrographs are captured using a $20 \times$ objective lens $(\mathrm{NA}=0.45)$. 

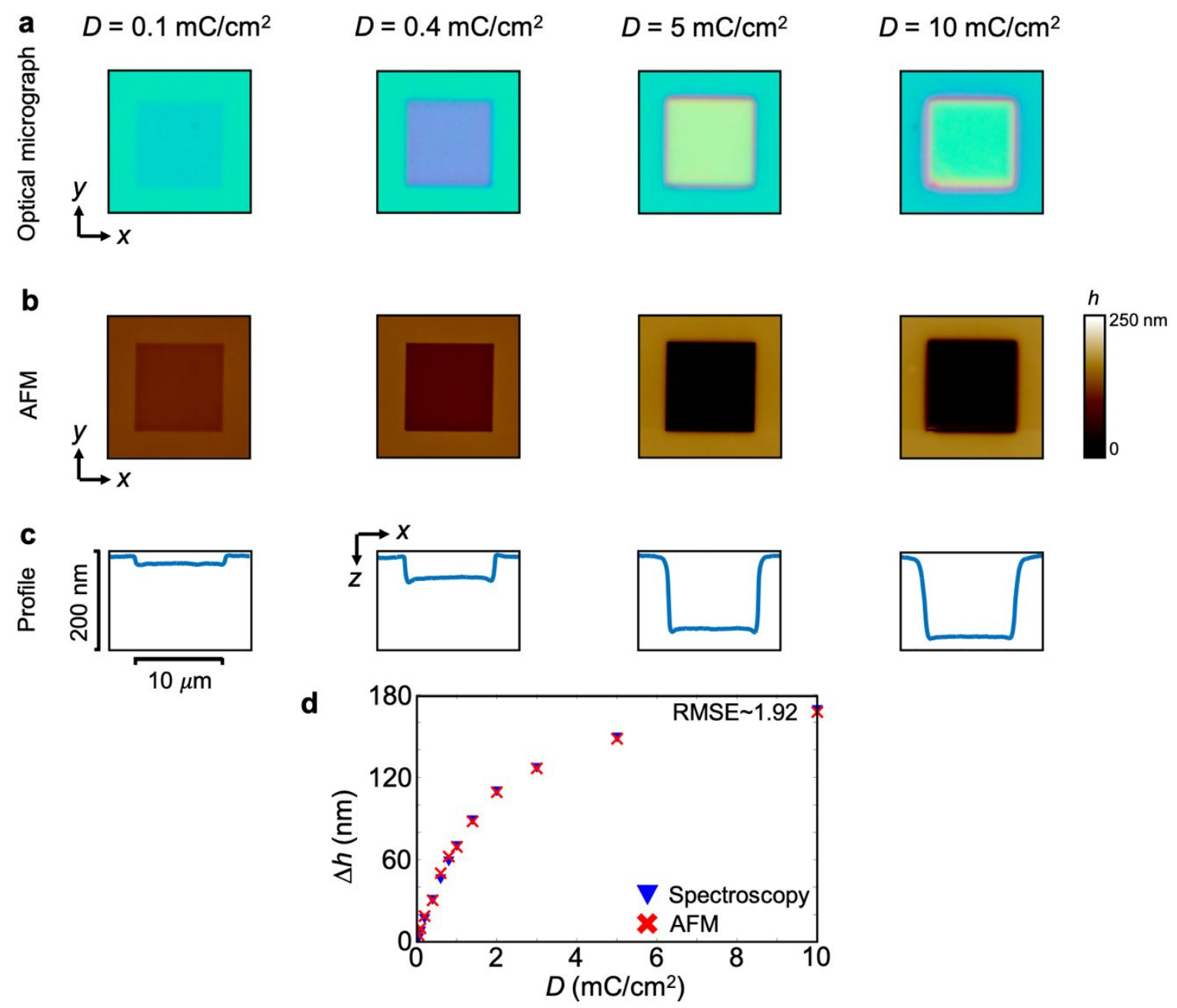

Figure S3. (a) Optical micrographs of square arrays with various exposure durations. (b) Corresponding AFM micrographs. (c) Profile of the square arrays. (a) Optical micrographs are captured using a $20 \times$ objective lens $(\mathrm{NA}=0.45)$. AFM measurements were done in tapping mode. (d) Shrinkage values obtained by AFM vs. spectroscopic measurements (simulation data was fitted to spectroscopic values to determine the thickness). 
a

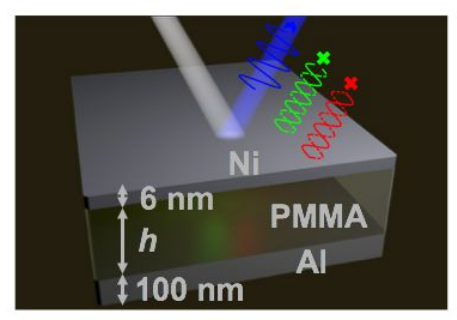

b

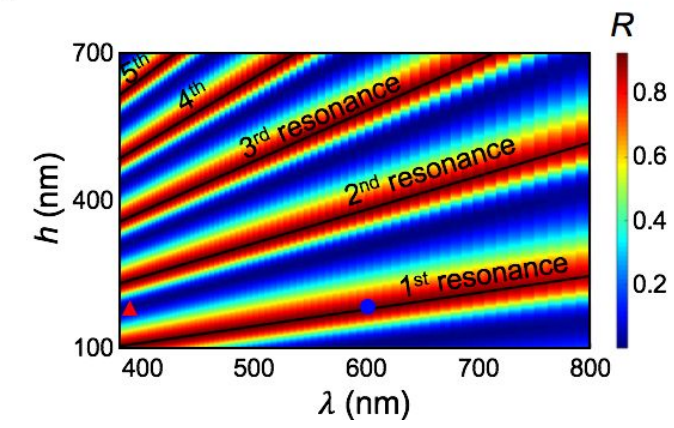

Figure S4. (a) Schematic of the FP cavity. (b) Simulated reflectance map for PMMA thicknesses of 100 to $700 \mathrm{~nm}$. The reflectance minimum $(\lambda=390 \mathrm{~nm})$ and maximum $(\lambda=600$ $\mathrm{nm}$ ) for a PMMA thickness of $h=180 \mathrm{~nm}$ are highlighted on the reflectance map. 


\section{Pixel size effect on color}

a

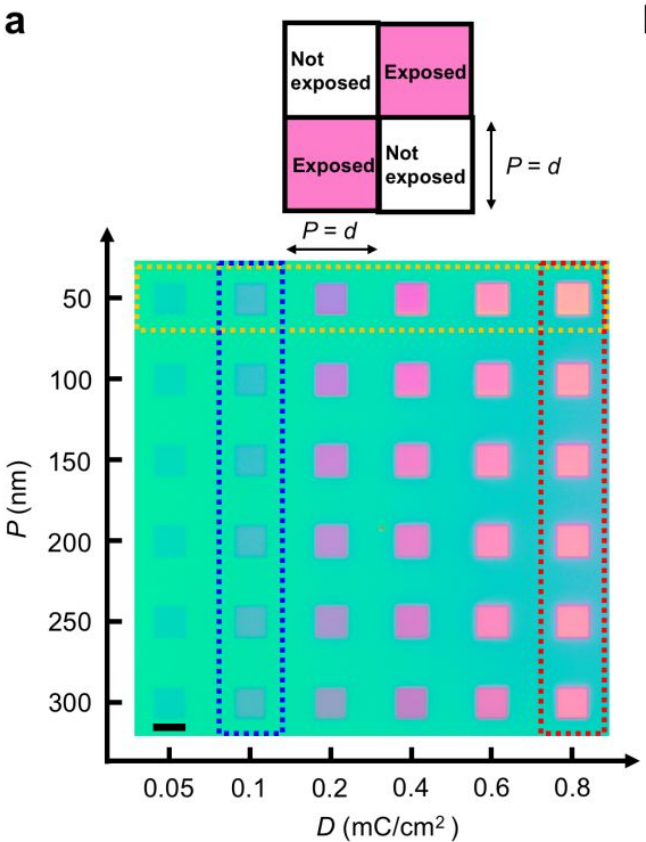

b
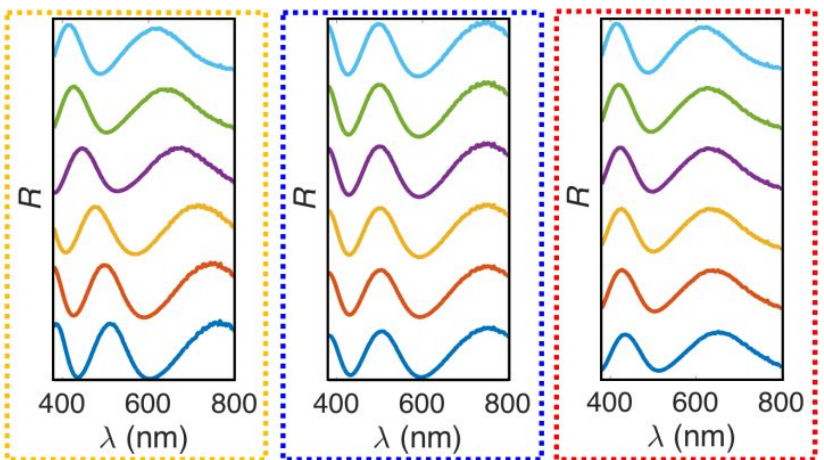

Figure S5. (a) Optical micrographs of square arrays of checkerboard pixels with various pixel sizes and doses. Pixel schematic is also shown. (b) corresponding reflectance spectra as highlighted by yellow, blue, and red dashed boxes. Optical micrographs are captured using a $20 \times$ objective lens $(\mathrm{NA}=0.45)$ and scale bar is $10 \mu \mathrm{m}$. 
a

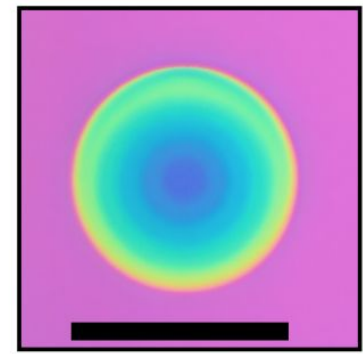

b

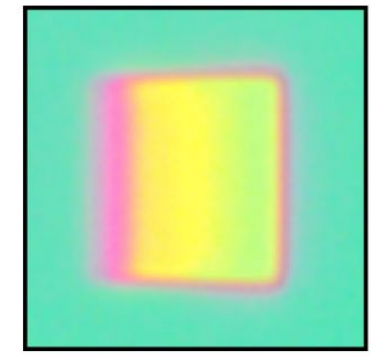

Figure S6. (a) A multistep disk and (b) a square with variable height. Optical micrographs are captured using a $20 \times$ objective lens $(\mathrm{NA}=0.45)$ and scale bar is $10 \mu \mathrm{m}$. 
Height map obtained using image analysis and those measured by AFM and the corresponding height map difference

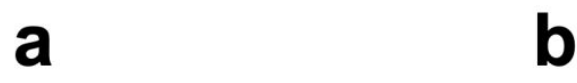

Image analysis

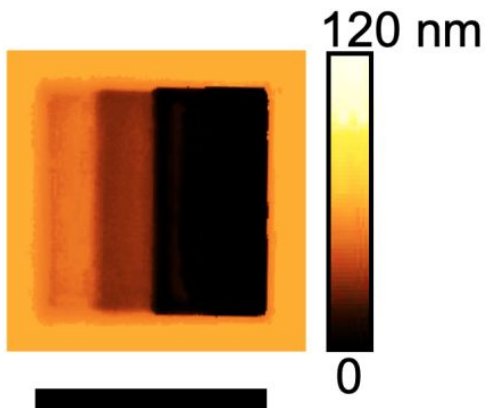

b

AFM

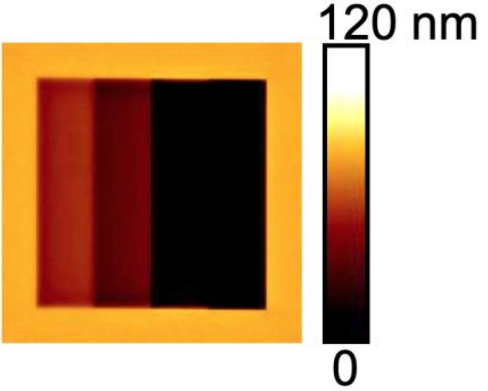

C

\section{Error}

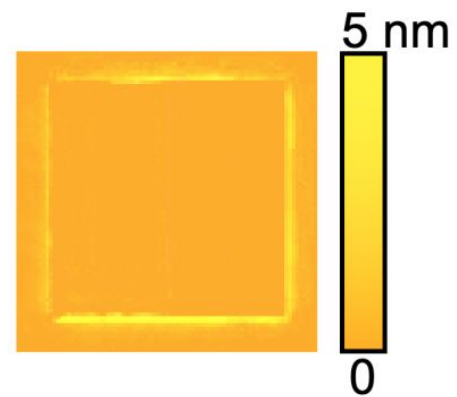

Figure S7. (a) A height map obtained by image analysis, (b) measured by AFM, and (c) the corresponding height difference (error). The scale bar in (a) is $10 \mu \mathrm{m}$. 
Direct fabrication of blazed and binary gratings. The method is not limited to patterning FP cavities but can be employed to realize any pattern with variable heights in PMMA. Here we fabricated blazed and binary diffraction gratings to disperse light. Since the blazed grating has a variable-height profile, it cannot be fabricated by conventional E-beam resists in a single step i.e., resist development step is still necessary. More importantly, the grayscale lithography in PMMA tends to cause severe roughness for the low dose regions. The binary and blazed gratings with rectangular and sawtooth grooves are illustrated in Figure S9a. The Optical and AFM micrograph along with gratings grooves profiles are shown in Figure S8b-d for binary (top) and blazed (bottom) gratings. As can be seen from the AFM profile in Figure S9c, the sawtooth profile can be achieved in a single step. 
Figure S9e shows the reflectance spectra for $x$ and $y$-polarized lights at normal incidence for binary (top) and blazed (bottom) gratings. The diffraction efficiency eta is shown for $x$ polarized light incident at $30^{\circ}$ and $20^{\circ}$ for binary (top) and blazed (bottom) gratings respectively. As can be seen, the reflectance spectra for $x$-polarized and $y$-polarized lights are considerably different, which is expected. The diffraction efficiency of the binary glazing has a maximum at around $\lambda=500 \mathrm{~nm}$, which is why green color is dominant in the optical image in Figure S9f (top). The maximum efficiency for the blazed gratings, on the other hand, has a maximum around the blue region, which is seen in the optical image in Figure S9f (bottom). It should be highlighted that the diffraction intensity appears to be lower in Figure S9f (bottom) compared to the image in Figure S9f (top) which is attributed to the lower diffraction efficiency of the blazed grating.

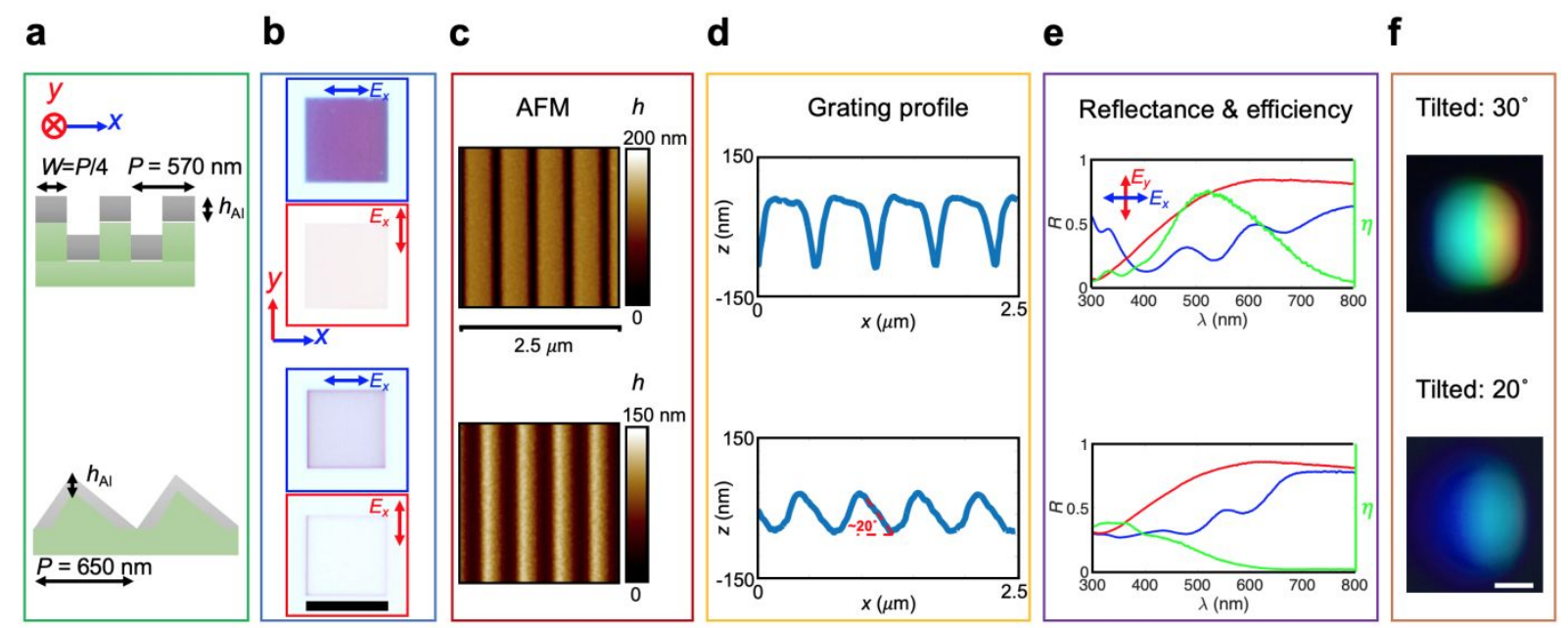

Figure S8. (a) Schematics of binary (top) and blazed (bottom) gratings. (b) Optical micrograph of binary (top) and blazed (bottom) gratings for a $x$ and $y$-polarized incident light source. (c) AFM micrographs of the binary (top) and sawtooth (bottom) gratings. (d) The binary (top) and sawtooth (bottom) profiles (e) Reflectance spectra for $x$ and $y$-polarized lights at normal incidence for binary (top) and blazed (bottom) gratings. The diffraction efficiency $\boldsymbol{\eta}$ is shown for an $x$-polarized light at incidence angles of $30^{\circ}$ and $20^{\circ}$ for binary (top) and blazed (bottom) gratings, respectively. (f) Tilted-view bright-field optical micrographs of binary (top) and blazed (bottom) gratings. The scale bars in (b) and (f) are $30 \mu \mathrm{m}$ and $10 \mu \mathrm{m}$, respectively. Optical micrographs are captured using a $10 \times$ objective lens. 


\section{Nanogrids}

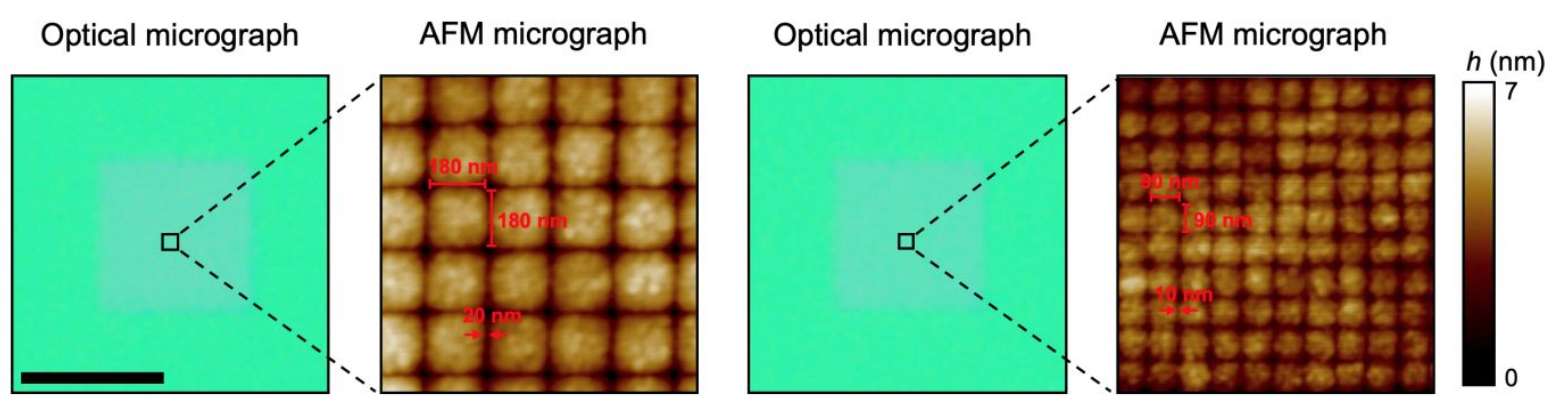

Figure S9. Optical and AFM micrographs of square grids with $180 \mathrm{~nm}$ and $90 \mathrm{~nm}$ line intervals realized by an E-beam dose of $0.05 \mathrm{mC} \mathrm{cm}^{-2}$. The scale bar is $10 \mu \mathrm{m}$. Optical micrographs are captured by a $20 \times$ objective lens (NA: 0.45 ). 


\section{AFM micrograph}

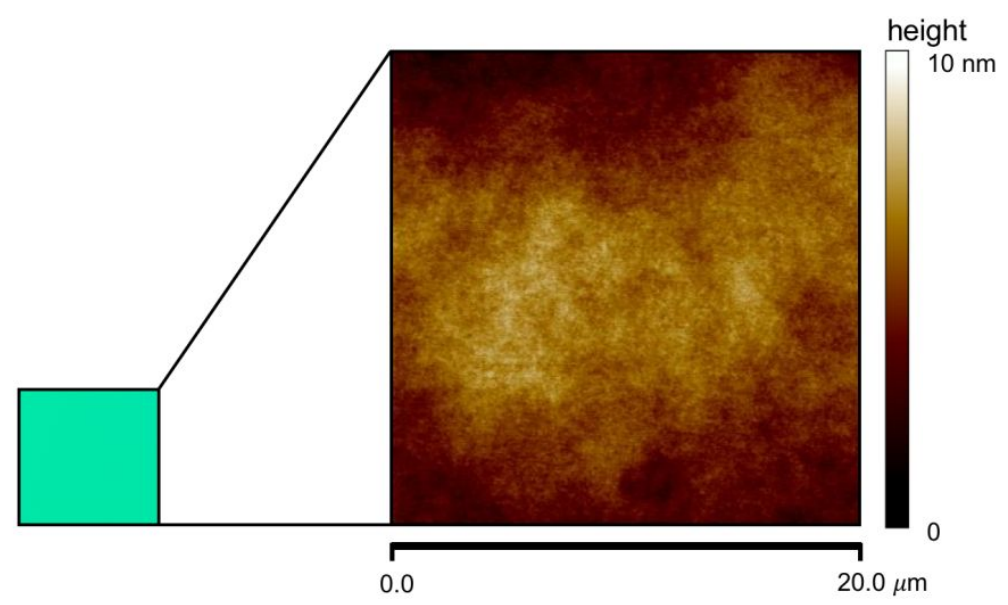

surface roughness $=1.23 \mathrm{~nm}$

Figure S10. Optical micrograph and surface morphology of a sample with $h=500 \mathrm{~nm}$. AFM measurement was done in tapping mode. Optical micrographs captured using a $\times 20$ objective lens $(\mathrm{NA}=0.45)$. 
Double Gaussian proximity effect function (PEF): Literature values vs. derived values

Values in Table S1 and Table S2 are calculated based on the following double Gaussian and double Gaussian plus exponential functions that model the electron beam exposure profile:

$$
\begin{aligned}
& f(r)=a_{1} \exp \left(\frac{-r^{2}}{b_{1}^{2}}\right)+a_{2} \exp \left(\frac{-r^{2}}{b_{2}^{2}}\right) \\
& f(r)=a_{1} \exp \left(\frac{-r^{2}}{b_{1}^{2}}\right)+a_{3} \exp \left(\frac{-r}{b_{3}}\right)+a_{2} \exp \left(\frac{-r^{2}}{b_{2}^{2}}\right)
\end{aligned}
$$

Equation 1

Equation 2

Table S1 Coefficients of PEF (double Gaussian) reported in the literature vs. derived values

\begin{tabular}{ccccccc}
\hline $\begin{array}{c}\text { PEF } \\
\text { Coefficients }\end{array}$ & $\begin{array}{c}\text { This work } \\
\mathbf{S i}, \mathbf{1 0 0} \mathbf{~ k e V}\end{array}$ & $\begin{array}{c}\mathbf{S i} \\
\mathbf{1 0 0} \mathbf{k e V}^{1}\end{array}$ & $\begin{array}{c}\mathbf{S i} \\
\mathbf{1 0 0} \mathbf{k e V}^{1]}\end{array}$ & $\begin{array}{c}\mathbf{S i} \\
\mathbf{1 0 0} \mathbf{k e V}^{2}\end{array}$ & $\begin{array}{c}\mathbf{S i} \\
\mathbf{1 0 0} \mathbf{k e V}^{3}\end{array}$ & $\begin{array}{c}\mathbf{S i} \\
\mathbf{1 0 0}_{\mathbf{~ k e V}}\end{array}$ \\
\hline $\boldsymbol{a}_{\mathbf{1}}$ & 1 & 1 & 1 & - & - & - \\
$\boldsymbol{a}_{\mathbf{2}}$ & $5.19 \mathrm{e}-6$ & $1.25 \mathrm{e}-6$ & $5.0 \mathrm{e}-6$ & - & - & - \\
$\boldsymbol{b}_{\mathbf{1}}$ & $29.2 \mathrm{~nm}$ & $40 \mathrm{~nm}$ & $80 \mathrm{~nm}$ & $80 \mathrm{~nm}$ & - & - \\
$\boldsymbol{b}_{\mathbf{2}}$ & $28.66 \mu \mathrm{m}$ & $32 \mu \mathrm{m}$ & $32 \mu \mathrm{m}$ & $26 \mu \mathrm{m}$ & $33.7 \mu \mathrm{m}$ & $27 \mu \mathrm{m}$ \\
\hline
\end{tabular}

Table S2 Coefficients of PEF (double Gaussian plus exponential) reported in the literature vs. derived values

\begin{tabular}{ccc}
\hline $\begin{array}{c}\text { PEF } \\
\text { Coefficients }\end{array}$ & This work & $\mathbf{S i}$ \\
$\mathbf{S i}, \mathbf{1 0 0} \mathbf{k e V}$ & $100 \mathbf{k e V}^{1]}$ \\
\hline $\boldsymbol{a}_{\mathbf{1}}$ & $1.26 \mathrm{e}-5$ & 1 \\
$\boldsymbol{a}_{\mathbf{2}}$ & $9.2 \mathrm{e}-3$ & $5.0 \mathrm{e}-6$ \\
$\boldsymbol{a}_{\mathbf{3}}$ & $21.7 \mathrm{~nm}$ & - \\
$\boldsymbol{b}_{\mathbf{1}}$ & $35 \mu \mathrm{m}$ & $40 \mathrm{~nm}$ \\
$\boldsymbol{b}_{\mathbf{2}}$ & $307 \mathrm{~nm}$ & $32 \mu \mathrm{m}$ \\
$\boldsymbol{b}_{\mathbf{3}}$ & & $500 \mathrm{~nm}$
\end{tabular}




\section{References}

(1) Koba, F.; Yamashita, H.; Arimoto, H. Highly Accurate Proximity Effect Correction for 100 KV Electron Projection Lithography. Japanese J. Appl. Physics, Part 1 Regul. Pap. Short Notes Rev. Pap. 2005, 44 (7 B), 5590-5594.

(2) Rooks, M.; Belic, N.; Kratschmer, E.; Viswanathan, R. Experimental Optimization of the Electron-Beam Proximity Effect Forward Scattering Parameter. J. Vac. Sci. Technol. B Microelectron. Nanom. Struct. 2006, 23 (6), 2769.

(3) Boere, E.; van der Drift, E.; Romijn, J.; Rousseeuw, B. Experimental Study on Proximity Effects in High Voltage E-Beam Lithography. Microelectron. Eng. 1990, 11 (1-4), 351-354.

(4) Anderson, E. H.; Olynick, D. L.; Chao, W.; Harteneck, B.; Veklerov, E. Influence of Sub-100 Nm Scattering on High-Energy Electron Beam Lithography. J. Vac. Sci. Technol. B Microelectron. Nanom. Struct. 2002, 19 (6), 2504. 